\title{
Which type of post and core system should you use?
}

\author{
What is the most effective post and core system to use in the restoration of \\ endodontically-treated teeth?
}

\author{
Bolla M, Muller-Bolla M, Borg C, Lupi-Pegurier L, Laplanche \\ $\mathbf{O}$, Leforestier E. Root canal posts for the restoration of root filled \\ teeth. Cochrane Database of Systematic Reviews 2007; issue 1
}

Data sources Articles were sourced using the Cochrane Central Register of Controlled Trials (CENTRAL), Medline, Scopus (abstract and citation database; www.scopus.com), Embase and the reference lists of articles and dental conference proceedings. Researchers in the field and manufacturers were also contacted.

Study selection Randomised controlled clinical trials (RCT) or quasiRCT were selected if they compared failures in endodontically-treated permanent teeth with different types of post.

Data extraction and synthesis Two review authors independently assessed the quality of trials and extracted data. Study authors were contacted for additional information.

Results Two trials involving 317 participants were included, but only one of them (which had 200 participants) compared metal with nonmetal posts. The other answered the secondary objective. The risk of failure was greater with metal-cast posts (nine out of 98 metal posts failed) than with carbon fibre posts [using which, none out of 97 failed; risk ratio (RR), 0.05 ; $95 \%$ confidence interval $(\mathrm{Cl}), 0.00-0.90$ ] but the study was at high risk of bias. Thus, even though fewer failures occurred when using nonmetal posts, the evidence is unreliable.

Conclusions This review could not specify which type of post and core system should be used when two or three dentine walls remain. More RCT are needed to confirm whether fibre-reinforced post and core systems are superior, and to clarify the influence of the remaining tooth structure on the treatment outcome using different post and core systems. Well-defined inclusion criteria focusing on the number of dentine walls (two or three) should be used.

\section{Commentary}

This Cochrane review kills two birds with one stone. First, it reports on a very important and common area of dental practice. Second, it highlights how, for all the dental literature published on restoring endodontically treated teeth with posts and cores, there is only one RCT addressing the primary objective of this review, and that has a high risk of bias.

The review is fairly simple and easy to read. The primary objective was clearly and plainly stated: to compare the clinical failure rates of different types of posts used for the restoration of endodontically treated teeth. The secondary objective, however, was phrased more obliquely, making it less straightforward for the general dentist to fully understand the aims. The search strategy was comprehensive enough, with regards to the databases and languages, to detect all potentially relevant studies. The inclusion and exclusion criteria were clear and easy to follow. The discussion and conclusions were short and sweet, putting everything into perspective and wrapping up all the details.

The restoration of endodontically treated teeth is a very common procedure in dentistry. Based on the results of this review, one would question the evidence basis for the selection of the post and core systems that are used in the hundreds, if not thousands, of endodontically treated teeth restored everyday. The previously available evidence of classical (narrative-type) reviews, ${ }^{1,2}$ their updates ${ }^{3,4}$ and in-vitro studies have been, and are still, used as evidence for or against the different post and core systems and techniques. These reviews are not based on high-quality evidence, however, as this Cochrane review confirms. In addition, no reliable guidelines exist to determine which post and core system should be used in relation to the amount of remaining tooth structure. Although these lower levels of evidence can provide cautious guidance for us in our treatment decisions, we certainly need to conduct more high quality RCT to identify which post and core system is superior and thus provide better treatment for our patients.

\section{Practice point}

The question of which post and core system has the lowest failure rate in the treatment of endodontically treated has yet to be properly answered.

\section{Asim Al-Ansari \\ Prosthodontics and Dental Public Health, Dental Department, Armed Forces Hospital, Dhahran, Saudi Arabia \\ 1. Morgano SM, Milot P. Clinical success of cast metal posts and cores. J Prosthet Dent 1993; 70:11-16. \\ 2. Goodacre C], Spolnik KJ. The prosthodontic management of endodontically treated teeth: a literature review. Part I. Success and failure data, treatment concepts. J Prosthodont 1994; 3:243-250. \\ 3. Morgano SM, Rodrigues AH, Sabrosa CE. Restoration of endodontically treated teeth. Dent Clin N Am 2004; 48:397-416. \\ 4. Goodacre CJ. Five factors to be considered when restoring endodontically treated teeth. Pract Proced Aesthet Dent 2004; 16:457-462.}

Evidence-Based Dentistry (2007) 8, 5-6. doi:10.1038/sj.ebd.6400489 